\begin{tabular}{|c|c|c|c|c|c|c|c|c|}
\hline \multirow{2}{*}{2} & 0 & $\mathbf{P}$ & 8 & 1 & $\mathbf{D}$ & \multirow{2}{*}{$\begin{array}{r}\text { Dispatch: } 30.12 .10 \\
\text { Author Received: }\end{array}$} & \multirow{2}{*}{ Journal: OP0 } & \multirow{2}{*}{ CE: Chandra } \\
\hline & Journal & Name & Manuscrip & t No. & & & & \\
\hline
\end{tabular}

\title{
A comparison of the NCT Reichert R7 with Goldmann applanation tonometry and the Reichert ocular response analyzer
}

Jorge Jorge, José M González-Méijome, António Queirós, Paulo Fernandes and José A Diaz-Rey

Clinical \& Experimental Optometry Research Lab, Center of Physics, School of Science, University of Minho, Braga, Portugal

Citation information: Jorge J, González-Méijome JM, Queirós A, Fernandes P \& Diaz-Rey JA. A comparison of the NCT Reichert R7 with Goldmann applanation tonometry and the Reichert ocular response analyzer. Ophthalmic Physiol Opt; doi: 10.1111/j.1475-1313.2010.00817.x

Keywords: Goldmann applanation

tonometer, intraocular pressure, non-contact tonometer, ocular response analyzer, Reichert R7

Correspondence: Jorge Jorge

E-mail address: Jorge@fisica.uminho.pt

Received: 6 May 2010; Accepted: 16 December 2010

\begin{abstract}
Purpose: The aim of this study was to evaluate the accuracy of intraocular pressure (IOP) values from the new non-contact tonometer (NCT) Reichert R7 by comparing results with those from Goldmann applanation tonometry (GAT) and the Reichert Ocular Response Analyzer (ORA). Other ocular dimensions were assessed to evaluate their potential influence on the IOP values obtained.

Methods: Ninety two right eyes from 92 adults aged between 21 and 59 years (mean $34.9 \pm 11.7$ years) were enrolled in this study. IOP was measured with R7, ORA and GAT. All measurements were taken between 14:00 and 16:00 in the afternoon. Corneal resistance factor (CRF) and corneal hysteresis were measured with the Reichert ORA. The spherical equivalent refractive error was obtained as using an open field auto-refractor (WAM5500; Grand Seiko) and corneal curvature, anterior chamber depth, corneal diameter and axial length were assessed with an optical coherence biometer (IOL Master; Zeiss Meditec, CA, USA).

Results: The mean values for IOP measurements were $15.20 \pm 3.37 \mathrm{mmHg}$ (R7), $13.49 \pm 3.55 \mathrm{mmHg}$ (GAT), $15.01 \pm 3.38 \mathrm{mmHg}$ (ORA IOPcc) and $14.44 \pm 3.47 \mathrm{mmHg}$ (ORA IOPg). With the exception of the CRF $(\rho=0.72$ $p<0.001)$ the correlations between ocular parameters and IOP obtained with the R7 were neither statistically nor clinically significant.

Conclusions: The new NCT, R7 overestimated the IOP compared with GAT in normal, healthy eyes by about $1.7 \mathrm{mmHg}$ on average ( $95 \%$ confidence range of approximately -2 to $+6 \mathrm{mmHg}$ ). The measures provided by the $\mathrm{R} 7$ were significantly influenced by the stiffness of the corneal tissue as measured by the ORA CRF value but not by other dimensional parameters of the eye.
\end{abstract}

\section{Introduction}

The use of non-contact tonometry (NCT) is now well established in clinical practice, mainly due to its important role in glaucoma case finding, since intraocular pressure (IOP) is perhaps the most important risk factor for the development of the disease. Moreover, reducing IOP is one of the first and most important steps in current management of glaucoma. Goldmann applanation tonometry (GAT) remains the gold standard technique for assessing IOP. However, as NCTs do not require corneal anesthesia and do not make direct contact with the cornea, they reduce the risk of corneal epithelial abrasion or cross infection between patients and they can be easily measured by trained clinical assistants. NCTs are particularly useful for IOP measurement in those countries (all European Union countries with the exception of the United Kingdom, Ireland and the Netherlands) where diagnostic drugs cannot presently be used by optometrists.

NCTs have been used to measure IOP in the clinical setting since Grolman introduced the first device in 1972s. ${ }^{1}$ Since then improvements in technology have made possible the design and manufacture of increasingly accurate and reliable NCTs. ${ }^{2-6}$ The Reichert 7 (R7) NCT 